EPJ Web of Conferences 17, 14001 (2011)

DOI: $10.1051 /$ epjconf/20111714001

(C) Owned by the authors, published by EDP Sciences, 2011

\title{
Recent results from HELIOS
}

\author{
B. B. Back ${ }^{\mathrm{a}}$ for the HELIOS Collaboration ${ }^{\mathrm{b}}$ \\ Physics Division, Argonne National Laboratory, Argonne, IL 60439, USA
}

\begin{abstract}
Transfer and inelastic scattering reactions using light-ion beams and stable or long-lived targets have traditionally provided detailed information on the structure of nuclei near the line of beta stability. Such studies can now be extended to nuclei away from the line of $\beta$-stability as radioactive beams are becoming available at a number of facilities around the world, including the CARIBU facility at Argonne. These measurements must, however, be carried out in inverse kinematics, resulting in a loss of the effective experimental resolution when conventional detection schemes are employed. The HELIOS spectrometer is based on a new concept, that is especially well suited for such studies by reducing the resolution problem, providing simple particle identification, and giving high detection efficiency with moderate Si detector area. In this talk, the HELIOS concept and results from the first series of experiments will be presented.
\end{abstract}

\section{Introduction}

The Nuclear Shell Model by Mayer and Jensen [1] has provided impetus for the study of single-particle orbits in excited nuclei throughout the nuclear chart. Much experimental work at laboratories and universities throughout the world resulted in detailed information on the single-particle structure, strength, and spin-parity assignments of excited states in both spherical nuclei at or near the closed shells and the deformed nuclei that fall in-between them. A rapid development of experimental facilities and techniques took place over the same period at many research centers. Precision beams of light particles became available from Van de Graaff Tandem accelerators of ever increasing terminal voltage, and the development and implementation of high-resolution magnetic (Bruekner, Enge Split-Pole, Elbek, Multigap, and Q3D) spectrometers were the tools needed to carry out experiments with the high energy resolution that is required to measure the excitations in nuclei across the periodic table. In addition, the discovery of direct nuclear reactions by Butler [2], its elaboration in terms of the Distorted Wave Born Approximation [3,4], and the signif-

\footnotetext{
a e-mail: back@anl.gov

b B.B.Back*, S.I.Baker*, J.A.Clark*, C.M.Deibel*,\%, B.J.DiGiovine*, S.J.Freeman ${ }^{+}$, N.J.Goodman\#, C.R. Hoffman*, B.P.Kay*, H.Y.Lee*, J.C.Lighthall $^{*}, *$, C.J.Lister*, S.T.Marley\#,*, P.Mueller*, R.Pardo*, K.E.Rehm*, A.M.Rogers*, J.Rohrer*, J.P.Schiffer*, D.V.Shetty\#, J.Snyder", A.H.Wuosmaa ${ }^{\#}$ - *Argonne National Laboratory, Argonne, IL 60439, USA - \#Western Michigan University, Kalamazoo, MI 49008, USA ${ }^{+}$University of Manchester, Manchester M13 9PL, UK -

${ }^{\%}$ Joint Inst. for Nucl. Astroph. Michigan State University, East Lansing, MI 48824, USA
}

icance of spectroscopic overlaps by French and Macfarlane [5] established elegant connections between the theoretical concepts and the experimental observables. The extension of the understanding of single-particle structure to deformed nuclei by S.G. Nilsson [6] gave a more complete picture of all accessible nuclei.

The literature contains information on virtually all nuclei that can be reached with light charged particle reactions on stable or long-lived targets, but the record is not always complete and/or the data analysis method for a series of isotopes in a region may not have been sufficiently uniform to study systematic trends. However, recent systematic measurements of the single-particle strength distribution have been carried out in the $\mathrm{Sn}$ region [7] and in $\mathrm{N}=82$ nuclei [8].

\section{Using radioactive beams}

In recent years, radioactive heavy-ion beams, away from the line of $\beta$-stability, have become available for studies of nuclear structure. High-energy beams produced by projectile fragmentation can be used in nucleon knock-out reactions to obtain information about particle orbits below the Fermi surface. In order to conduct detailed studies of single particle states above the Fermi surface, beams at energies comparable to the Coulomb barrier are needed. Such beams can be provided as secondary beams produced in flight [9] or as re-accelerated radioactive ions. Radioactive-ion production methods include ions extracted from a primary ${ }^{252} \mathrm{Cf}$ fission source at the CARIBU facility at Argonne [10] or from targets bombarded with highintensity primary beams at HIE-ISOLDE at CERN [11], the HRIBF facility at Oak Ridge National Laboratory [12], at TRIUMF [13], and several other institu-

This is an Open Access article distributed under the terms of the Creative Commons Attribution-Noncommercial License 3.0, which permits unrestricted use, distribution, and reproduction in any noncommercial medium, provided the original work is properly cited. 
tions. In the near future, relatively intense, re-accelerated precision beams of this type will be provided at several new facilities, namely Spiral-2 at Ganil and FRIB at Michigan State University.

These new beams of radioactive nuclei will in many cases have sufficient intensity to allow studies of their single-particle structure via transfer reactions with light targets. The scientific thrust into these areas is driven by the need to validate the predictive power of present nuclear structure models, search for changes in the single particle structure far away from the line of $\beta$ stability where the Fermi levels for protons and neutrons are severely unbalanced. These studies are also important for Nuclear Astrophysics. The rapid neutroncapture $r$-process is, for example, strongly affected by the structure of neutron-rich nuclei near the $\mathrm{N}=82$ shell.

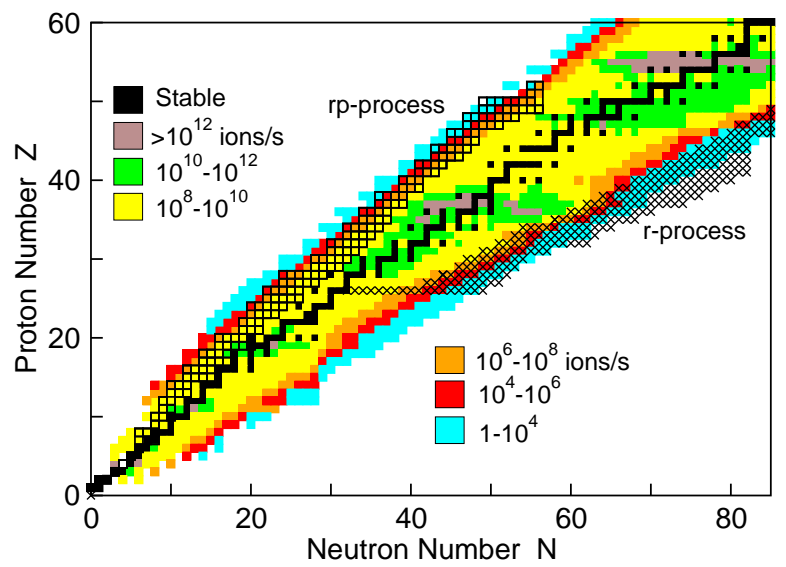

Fig. 1. This figure shows the intensities of re-accelerated beams that can be expected with $400 \mathrm{~kW} 200 \mathrm{MeV} / \mathrm{u}$ primary beams such as those at the planned FRIB facility. The map also indicates the expected location of the astrophysical $r p$ and $r$-processes.

Studies based on interactions with nuclei in a thin target, such as Coulomb excitation and transfer reaction, require beam intensities around $10^{3}$ to $10^{4}$ ions $/ \mathrm{s}$ to be feasible. As shown in Fig. 1 the region of the nuclear chart where beam intensities of sufficient magnitude are projected for a facility such as FRIB [15], is very substantial. The beam intensity at existing [13, 12,9] facilities as well as those that will start operation before FRIB $[16,11]$ will already allow for studies of less exotic nuclei as exemplified in a recent measurement of the single-neutron strength outside the doubly magic ${ }^{132} \mathrm{Sn}$ by Jones et al. [18].

\section{Challenges with inverse kinematics}

The many new exotic nuclei that are becoming available as re-accelerated beams require, however, that reactions are done in inverse kinematics, i.e. where the radioactive, heavy beam is incident onto a light (a)

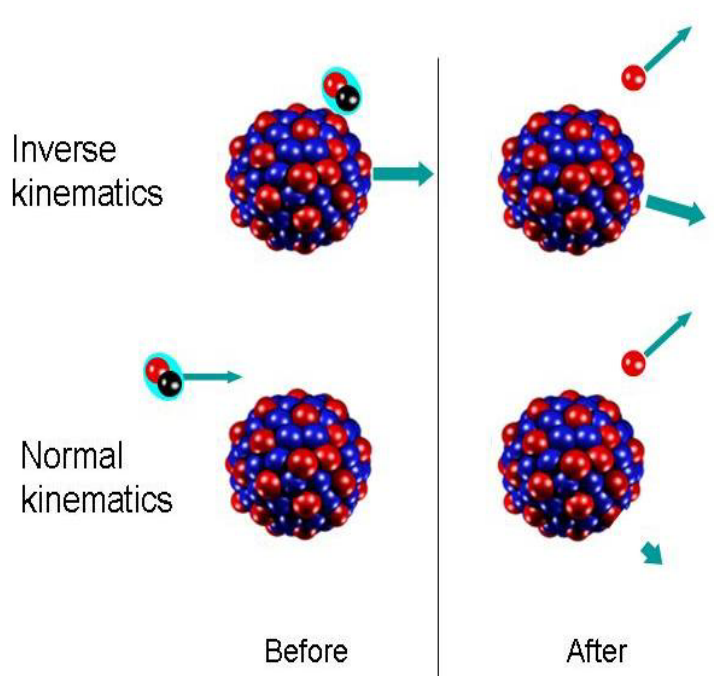

(b)

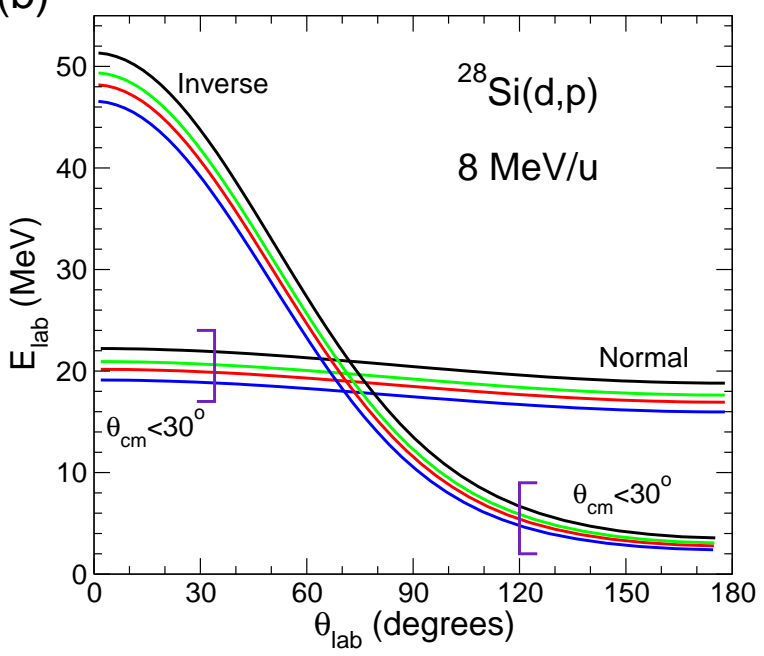

Fig. 2. Panel a: Illustration of "inverse" and "normal" kinematics reactions involving a simple neutron transfer. Panel b: Kinematic curves are shown for both "normal" and "inverse" kinematics for the ${ }^{28} \mathrm{Si}(\mathrm{d}, \mathrm{p})$ reaction with a beam energy of $8 \mathrm{MeV} / \mathrm{u}$ in both cases.

target (Fig. 2a). This requirement changes the experimental situation dramatically, because of the large center-of-mass velocity of the scattering system, which is similar to that of the beam. An example of the kinematic curves is given in Fig. $2 \mathrm{~b}$ for the reaction $\mathrm{d}\left({ }^{28} \mathrm{Si}, \mathrm{p}\right){ }^{29} \mathrm{Si}$ as compared with the "normal" kinematics for ${ }^{28} \mathrm{Si}(\mathrm{d}, \mathrm{p}){ }^{29} \mathrm{Si}$. In normal kinematics, the energy of the outgoing proton corresponding to the ground state and the three lowest excited states in ${ }^{29} \mathrm{Si}$, varies little as a function of laboratory angle. In contrast, inverse kinematics leads to a strong variation with angle, exceeding a factor of ten, which places severe demands on the detection. For the angles corresponding to forward scattering in the center-of-mass system, $\theta_{\text {c.m. }}<30^{\circ}$, that are of interest for angular momentum transfer determination, protons have only very low energies in the laboratory sytem, and the 


\section{FUSION11}

Table 1. Cyclotron periods of light charged particles in a magnetic field of strength $B=2$ Tesla.

\begin{tabular}{cc}
\hline Particle & $T_{c y c}(\mathrm{~ns})$ \\
\hline $\mathrm{p}$ & 32.8 \\
$\mathrm{~d}, \alpha$ & 65.6 \\
$\mathrm{t}$ & 98.4 \\
${ }^{3} \mathrm{He}$ & 49.2 \\
\hline
\end{tabular}

standard $\Delta \mathrm{E}$-E technique of particle identification becomes very problematic. In addition, the energy interval between the various excitations in the ${ }^{29} \mathrm{Si}$ nucleus is strongly reduced, by a factor $\sim 2.4$ relative to that observed in "normal" kinematics. The inverse kinematics situation therefore severely limits the number of nuclei that can be studied. It has, however, been demonstrated that systems with widely spaced excited states, including many light systems and nuclei near shell closures, can be explored with normal detector systems $[17,18]$. Nevertheless, it is clear that higher Q-value resolution is needed for nuclei in most of the new territory that is being made available with radioactive beam facilities, and that new techniques are required. This has led to the development at Argonne of a new type of spectrometer, the HELIical Orbit Spectrometer (HELIOS), that circumvents the main difficulties with inverse kinematics, as outlined above. In the following section I'll describe the principle of the HELIOS concept and discuss its implementation.

\section{Principle and implementation}

The HELIOS spectrometer is based on the fact that charged particles follow helical trajectories in a homogeneous magnetic field. If the magnetic field is aligned with the beam axis, see Fig. 3, the particles will return to the beam axis, which coincides with the magnetic axis of the spectrometer, provided that the magnetic field is strong enough to avoid collisions with the vacuum envelope of the magnet. The flight-time until it returns to the axis is the cyclotron period, $T_{c y c}=2 \pi m / q e B$, where $m / q$ is the charge to mass ratio of the particle and $B$ is the magnetic field strength. Note that the flight-time scales with $m / q$ of the particle so one obtains automatic particle identification for a range of light charged particles, such as protons, tritons, and ${ }^{3} \mathrm{He}$, by measuring this quantity. Deuterons and $\alpha$-particles have the same flight-time, but in many cases their flight-paths will not overlap. Cyclotron periods corresponding to a typical magnetic field strength of 2 Tesla are long enough that particle identification can be achieved rather comfortably with reasonable time resolution, see Table 1.

Particles emitted in the backward hemisphere return to the axis upstream of the target whereas forward emitted particles return downstream. Positionsensitive Si detector arrays may be placed at either lo-

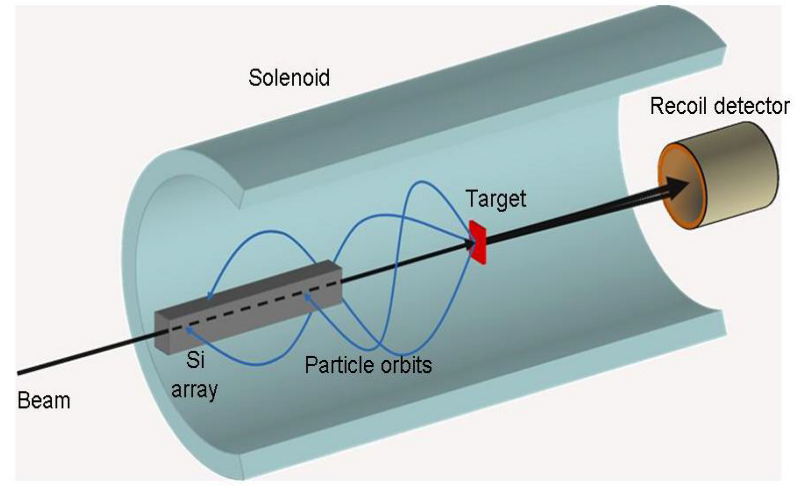

Fig. 3. Schematic illustration of the HELIOS concept. Beam enters from the left through a hollow tube that is supporting position sensitive Si detectors on the outside. Particles emitted from the target follow helical trajectories in the co-axial solenoidal magnetic field and return to the beam axis, some of them being intercepted by the Si detector array. Recoils may be detected in a detector located at very forward angles.

cation to register the time-of-arrival, $t$, energy, $E$, and longitudinal position, $z$, where the particle strikes the detector. The upstream array must be hollow to allow the beam to reach the target, whereas this requirement does not necessarily apply for a downstream array. However, in many cases it is advantageous to detect, in coincidence, the heavy beam-like recoils in detectors positioned at small angles, and in these cases a hollow array is called for.

There is a simple relation between $E, z$, and the center-of-mass energy of the particle, $E_{c m}$, namely

$$
E_{c . m .}=E+\frac{m}{2} V_{c m}^{2}-\frac{V_{c m} q e B}{2 \pi} z,
$$

where $V_{c m}$ is the laboratory velocity of the center-ofmass system, a quantity that is known for the reaction, (see Ref.[19]). It is important to note that, unlike the situation in a normal setup, the coefficient in front of $E$ is unity, which means that the measured level spacing is the same as what would be observed in the center-of-mass system. This feature effectively removes the detrimental effects of the inverse kinematics situation. The kinematic loci for different excitations in the nucleus are therefore straight lines for which the particle energy increases with $z$ (positive downstream) with a slope that scales with $V_{c m} q B$ as illustrated for the $\mathrm{d}\left({ }^{28} \mathrm{Si}, \mathrm{p}\right)$ reaction at $6 \mathrm{MeV} / \mathrm{u}$ in Fig. 4 .

The fact that no kinematic compression occurs when the particles are transported in a magnetic field arises because particles corresponding to different states emitted at the same laboratory angle returns to the beam axis (or the detector array) at different distances $z$ from the target as shown in Fig. 4.

A spectrometer based on this concept has recently been built at the ATLAS facility at Argonne National Laboratory. The main component is a large bore superconducting solenoid that was obtained from a de- 

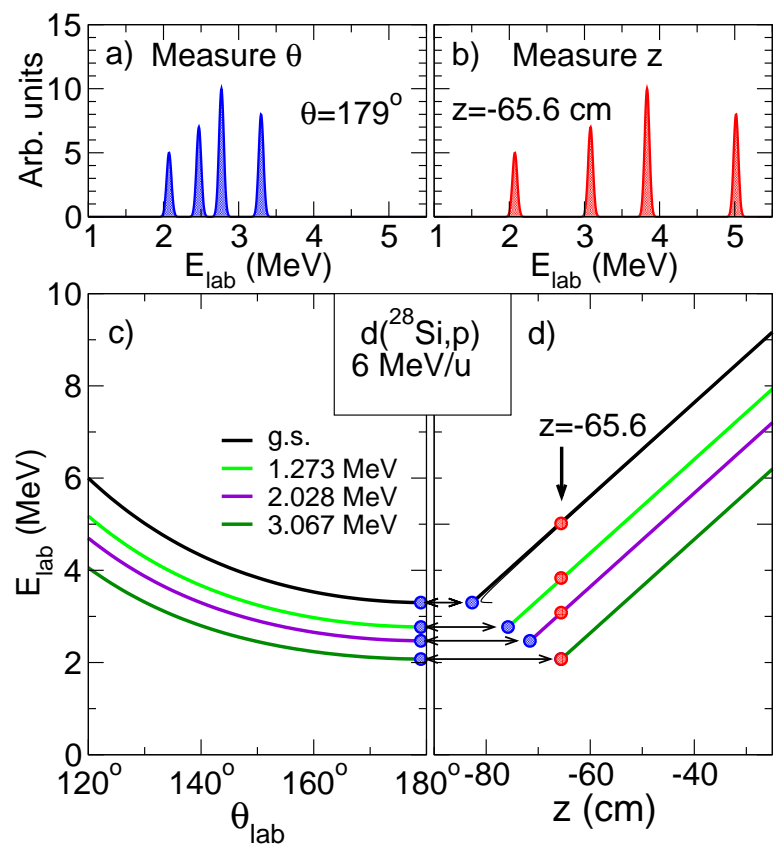

Fig. 4. Illustration of the improved Q-value resolution of the HELIOS concept exemplified by the $\mathrm{d}\left({ }^{28} \mathrm{Si}, \mathrm{p}\right)$ reaction populating the ground state and the first three excited states in ${ }^{29} \mathrm{Si}$. The ${ }^{28} \mathrm{Si}$ beam energy is $6 \mathrm{MeV} / \mathrm{u}$, and the assumed combined effects of target thickness and intrinsic energy resolution is $\Delta E=75 \mathrm{keV} F W H M$, for which the upper panels show simulated energy spectra for the emitted protons if measured either at a fixed angle $\theta=179^{\circ}$ (panel a) or a fixed z-position, $\mathrm{z}=-65.6 \mathrm{~cm}$ (panel b). Panels c and d, demonstrate that particles corresponding to different excitations emitted at a particular angle are intercepted by the $\mathrm{Si}$ detector at different z-positions when transported in a $2 \mathrm{~T}$, co-axial, homogenous field (blue points). By performing the energy measurement at a specific z-position instead (red points) the level spacing corresponding to that in the center-of-mass system is restored. The thin curve in panel $d$ for the g.s. gives the "hook" effect by intercepting the particles at a realistic radial distance of $1.4 \mathrm{~cm}$.

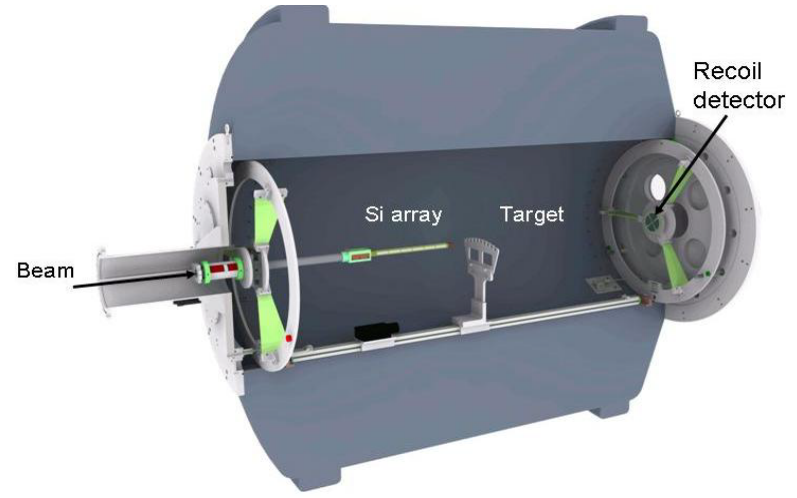

Fig. 5. Computer aided design rendering of the HELIOS spectrometer identifying the beam entrance, the Si detector array that is mounted onto an alignment ring, the target "fan" as well as of the recoil Si detector telescope. commissioned Magnetic Resonance Imaging scanner. This solenoid is able to generate a homogeneous magnetic field region over a cylindrical volume of diameter $90 \mathrm{~cm}$ and $100 \mathrm{~cm}$ in length. The strength of the magnetic field can be set up to 2.85 Tesla. The solenoid is mounted such that the magnetic field axis is coaxial with the beam. Fortunately, the end flanges, which were designed to support various RF and field gradient coils used for the MRI scanning, were such that they could be fitted with large vacuum covers that allowed the whole inside bore of the magnet to be evacuated by pumping through the $20 \mathrm{~cm}$ diam. beam-pipe. Support structures inside the magnet allow for the mounting of the hollow Si detector array at backward angles and a target "fan", both of which can slide longitudinally to allow for optimal coverage of the interesting region of particle emission (see Fig. 5). The design also allows for mounting of various detectors to register the coincident heavy recoils, shown in Fig. 5 as a Si detector telescope. Another recoil detector system consisting of an $\mathrm{x}-\mathrm{y}$ position sensitive PPAC backed by a co-axial Bragg curve spectrometer has been built, but this system has yet to be commissioned.

\section{Initial physics experiments}

The HELIOS spectrometer was successfully commissioned using the ${ }^{28} \mathrm{Si}(\mathrm{d}, \mathrm{p})$ reaction in August 2008 [20] and subsequently a number of physics experiments have been carried out using both stable and radioactive beams produced by the in-flight technique at ATLAS.

\section{$\mathbf{5 . 1}{ }^{12} \mathbf{B}(\mathbf{d}, \mathbf{p})$}

The first example is a study of the ${ }^{12} \mathrm{~B}(\mathrm{~d}, \mathrm{p})$ reaction populating excited states in ${ }^{13} \mathrm{~B}$ in an attempt to separate two positive-parity states at $\mathrm{E}_{\text {exc }}=3.48$ and 3.68 $\mathrm{MeV}$, which it had not been possible to separate in an earlier attempt with normal detectors [21]. In this experiment [22], the radioactive ${ }^{12} \mathrm{~B}$ beam was produced via a $(\mathrm{d}, \mathrm{p})$ reaction of the $81 \mathrm{MeV}$ primary ${ }^{11} \mathrm{~B}$ beam with an intensity of $50 \mathrm{pnA}$ in a cryogenic deuterium gas cell which resulted in a secondary ${ }^{12} \mathrm{~B}$ beam of approximately $10^{5}$ ions $/ \mathrm{s}$ at $75 \mathrm{MeV}$. The ${ }^{12} \mathrm{~B}$ ions were focused and separated from the primary beam using various beam-line elements [9] and brought into the HELIOS spectrometer to react with a $73 \mu \mathrm{g} / \mathrm{cm}^{2}$ thick $\mathrm{CD}_{2}$ target. A Si detector telescope covering the forward angular range $\theta_{l a b}=0.5^{\circ}-2.8^{\circ}$ consisting of 80 $\mu \mathrm{m}$ thick $\Delta \mathrm{E}$ and $500 \mu \mathrm{m}$ thick $\mathrm{E}$ detectors was used to measure ${ }^{13} \mathrm{~B}$ recoils in coincidence with the protons from the $(\mathrm{d}, \mathrm{p})$ reaction that were detected in the Si detector array positioned upstream of the target. The primary ${ }^{11} \mathrm{~B}$ beam was also used, at a reduced intensity, to measure the ${ }^{11} \mathrm{~B}(\mathrm{~d}, \mathrm{p})$ reaction to excited states in ${ }^{12} \mathrm{~B}$ at $\mathrm{E}_{e x c}=2.62$ and $3.39 \mathrm{MeV}$, which correspond to proton energies close to those for the ${ }^{13} \mathrm{~B}$ 


\section{FUSION11}

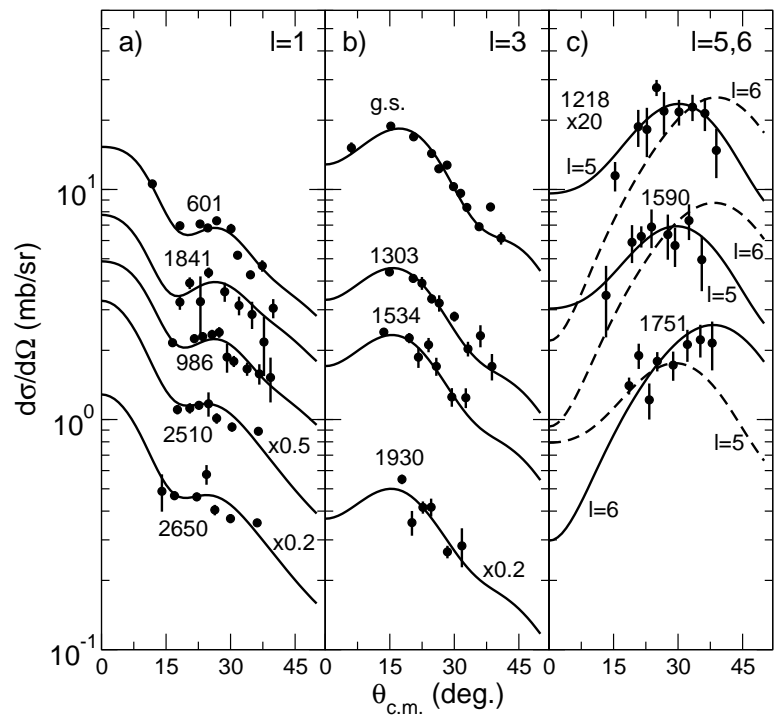

Fig. 6. The angular distributions for the ${ }^{11} \mathrm{~B}(\mathrm{~d}, \mathrm{p}){ }^{12} \mathrm{~B}$ reaction to the two states at $\mathrm{E}_{e x c}=2.62$ and $3.39 \mathrm{MeV}$ populated via $\mathrm{L}=0$ and $\mathrm{L}=2$ transfer are shown in the left panel. The known $\mathrm{L}=2$ transition to the $\mathrm{E}_{e x c}=3.39 \mathrm{MeV}$ state was used for normalization of the angular dependence of the detection efficiency. The red points are the HELIOS data for the $\mathrm{L}=2$ transition to the $3.39-\mathrm{MeV}$ state and the blue points are for the $\mathrm{L}=0,2.62-\mathrm{MeV}$ state. The solid and dashed curves are DWBA calculations with two different sets of parameters. On the right, the data for the ${ }^{12} \mathrm{~B}(\mathrm{~d}, \mathrm{p}){ }^{13} \mathrm{~B}$ doublet at 3.48 and $3.68 \mathrm{MeV}$ are shown with the same normalization. The conventions on the symbols and curves are the same as on the left-hand side.

doublet over the entire angular range. This measurement provided reference angular distributions used to normalize those measured for the ${ }^{13} \mathrm{~B}$ doublet and allowed us to determine the angular momentum transfer and spectroscopic factor for the two members of the $\mathrm{E}_{\text {exc }}=3.48$ and $3.68 \mathrm{MeV}$ doublet (see Fig. 6).

From these measurements it is clear that the 3.48 $\mathrm{MeV}$ member of the ${ }^{13} \mathrm{~B}$ doublet is populated by $\mathrm{L}=0$ transfer since it is very similar in shape to that of the $2.62 \mathrm{MeV}{ }^{12} \mathrm{~B}$ state, while the much weaker $3.68 \mathrm{MeV}$ member appears to be reached by $\mathrm{L}=2$ transfer. From these results and an analysis of the relative spectroscopic factors of the two states it appears that the $\mathrm{E}_{e x c}=3.48 \mathrm{MeV}$ and $3.68 \mathrm{MeV}$ states in ${ }^{13} \mathrm{~B}$ correspond to the expected $1 / 2^{+}$and $5 / 2^{+}$states in this nucleus, respectively. However, with this assignment, the spectroscopic factor for the $5 / 2^{+}$state is significantly weaker than predicted by theory.

\section{$5.2{ }^{15} \mathrm{C}(\mathrm{d}, \mathrm{p})$}

Recent results on electromagnetic transition rates between states in ${ }^{16} \mathrm{C}$ have led to the speculation that this nucleus has an exotic structure. A measurement of the $\mathrm{B}(\mathrm{E} 2)$ value for the $2_{1}^{+} \rightarrow 0^{+}$transition [24] using the Coulomb-nuclear interference method in ${ }^{208} \mathrm{~Pb}+{ }^{16} \mathrm{C}$ scattering thus obtained a $\mathrm{B}(\mathrm{E} 2)$ of $0.28 \pm 0.06$ W.U. leading to the suggestion that the $2_{1}^{+}$state is a pure valence neutron excitation. In addition, a direct lifetime measurement of the $2_{1}^{+} \rightarrow 0^{+}$transition using a recoil-shadow technique [25] obtained a result that corresponds to a $\mathrm{B}(\mathrm{E} 2)$-value of $0.26 \mathrm{~W}$.U. in agreement with the scattering result. In contrast with these results, a more recent measurement of the lifetime of the $2^{+}$state using a fusion reaction to populate states in the ${ }^{16} \mathrm{C}$ system obtains a result which corresponds to B(E2)-value of $1.73 \pm 0.30$ W.U. [26], consistent with theoretical expectations for even-even closed shell systems in this area and which does not require an "exotic" interpretation of the structure of the $2_{1}^{+}$state in ${ }^{16} \mathrm{C}$. With this background, it was felt that it might be advantageous to use a different experimental technique to explore the structure of the ${ }^{16} \mathrm{C}$ system.

Using an intense, $100 \mathrm{pnA}{ }^{14} \mathrm{C}$ beam of energy $133 \mathrm{MeV}$, a secondary ${ }^{15} \mathrm{C}$ beam was generated via the $(\mathrm{d}, \mathrm{p})$ reaction in a cryogenic gas cell in the ATLAS in-flight radioactive beam setup [9]. With this method we obtained a $123 \mathrm{MeV}{ }^{15} \mathrm{C}$-beam with intensity of $1-2 \times 10^{6}$ ions/s. This beam was transported to the HELIOS spectrometer impinging on a $110 \mu \mathrm{g} / \mathrm{cm}^{2}$ $\mathrm{CD}_{2}$ target. The detection of protons and coincident ${ }^{16} \mathrm{C}$ recoils was carried out in a manner similar to the ${ }^{12} \mathrm{~B}(\mathrm{~d}, \mathrm{p})$ experiment described above (see Ref. [23] for details).

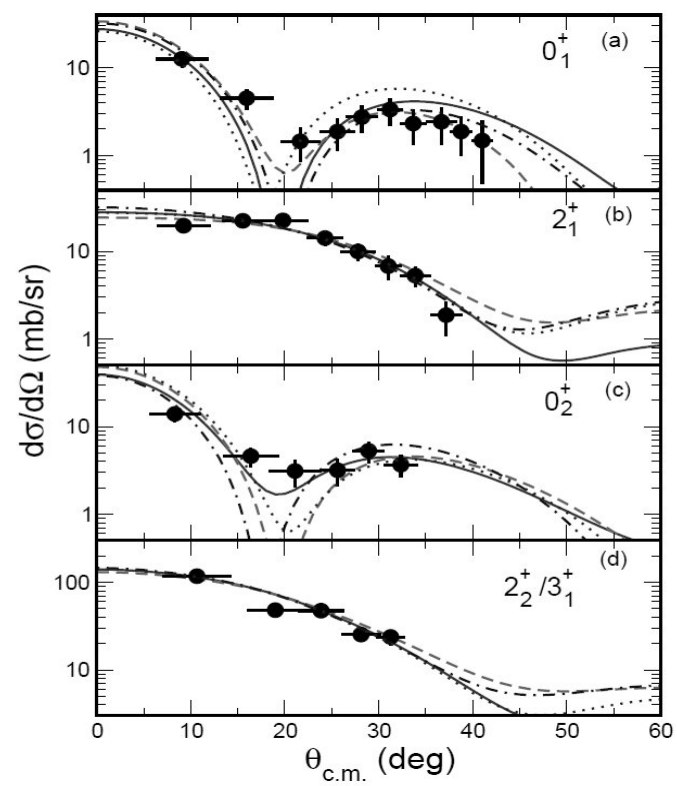

Fig. 7. The angular distributions for the ${ }^{15} \mathrm{C}(\mathrm{d}, \mathrm{p}){ }^{16} \mathrm{C}$ reaction to the $0^{+}$ground state (panel a), the first excited $2^{+}$state (panel b), the second excited $0^{+}$state (panel c), and the unresolved $2^{+} / 3^{+}$doublet (panel d) are shown along with DWBA calculations using four different Optical model potentials. The errors on the cross sections are statistical only and do not include contributions from systematic uncertainties (see Ref. [23] for details). 
The resulting angular distributions for scattering into the lowest excitations in ${ }^{16} \mathrm{C}$ are displayed in Fig. 7 along with DWBA calculations using four different Optical Model potentials. These comparisons lend strong support to the previously tentative assignment of $0^{+}$to the third excited state at $\mathrm{E}_{e x c}=3.017 \mathrm{MeV}$, since it is seen to be populated via an $\mathrm{L}=0$ transfer, as is the ground state. From the relative spectroscopic factors for the lowest-lying states one finds excellent agreement with shell-model calculations [27], which also correctly account for the latest measurement of the $\mathrm{B}\left(\mathrm{E} 2 ; 2_{1}^{+} \rightarrow 0^{+}\right)$transition matrix element [26]. Our ${ }^{15} \mathrm{C}(\mathrm{d}, \mathrm{p})$ results thus lend strong support to a standard Shell model description of this nucleus and appears to be at variance with an "exotic" picture of its structure.

\section{Summary}

Light-ion transfer-reaction studies on nuclei close to the line of $\beta$-stability, where such studies can be carried out with stable or long-lived targets in normal kinematics, have provided much of the existing nuclear structure information in these systems. However, such studies must be carried out in inverse kinematics to obtain similar information for the wide range of nuclear species that has or will become available at radioactive beam facilities. Using normal detection techniques leads to severe limitations in terms of $\mathrm{Q}$-value resolution in this situation. In this talk I have shown that the HELIOS concept, in which light charged particles are transported in a homogenous magnetic field to a hollow Si detector array located on the beam axis, resolves this particular problem and also allows for both high detection efficiency and easy particle identification. The Argonne HELIOS spectrometer was commissioned in August 2008 and the advantages of this technique were successfully demonstrated in a number of experiments. We have performed several studies of $(\mathrm{d}, \mathrm{p})$ reactions in inverse kinematics, including measurements of the $d\left({ }^{28} \mathrm{Si}, \mathrm{p}\right){ }^{29} \mathrm{Si}$ commissioning experiment as well as two published studies involving radioactive beams produced in-flight at the ATLAS facility. In the $\mathrm{d}\left({ }^{12} \mathrm{~B}, \mathrm{p}\right){ }^{13} \mathrm{~B}$ study, the improved Q-value resolution was crucial for separating two excited positive-parity states and studying their structure. The $d\left({ }^{15} \mathrm{C}, \mathrm{p}\right){ }^{16} \mathrm{C}$ experiment was the first study of this reaction and it helped validate the theoretical understanding of this nucleus in terms of the modern shell model. Additional experiments have been carried out for which the data analysis is ongoing. Several upgrades are planned for the spectrometer. These include a gas target for He induced reactions, a new, more efficient Si detector array, and a recoil PPAC and Bragg curve detector. With these further improvements we expect that the spectrometer will continue to support a very fruitful scientific program.

\section{Acknowledgments}

This work was supported by the U.S. Department of Energy, Office of Nuclear Physics under DE-AC0206CH11357 and DE-FG02-04ER41320, and NSF Grant No. PHY-08-22648.

\section{References}

1. M.G.Mayer, Phys. Rev. 74, 235 (1948); O. Haxel, J.H.D. Jensen, and H.E. Suess, Phys. Rev. 75, 1766 (1949).

2. S.T. Butler, Proc. Royal Soc. (London) A208, 559 (1951)

3. W. Tobocman and M.M. Kalos, Phys. Rev. 97, 132 (1955)

4. G.R. Satchler, Ann. Phys. 3, 275 (1958)

5. M. H. Macfarlane and J.B. French, Rev. Mod. Phys. 34, 41 (1960)

6. S.G.Nilsson, K. Danske Vidensk. Selsk. Mat-fys Medd., 29, 16 (1955)

7. J.P. Schiffer et al., Phys. Rev. Lett. 92, 162501 (2004)

8. B.P. Kay et al., Phys. Lett. B658, 216 (2008)

9. B. Harss et al., Rev. Sci. Instrum. 71, 380 (2000)

10. G. Savard et al., Nucl. Instr. Meth. B266, 4086 (2008)

11. http://hie-isolde.web.cern.ch/HIE-ISOLDE/

12. http://www.phy.ornl.gov/hribf

13. http://www.triumf.ca

14. http://www.frib.msu.edu

15. B.B.Back and C.L.Jiang, ANL internal report No. ANL-06/55 (2006)

16. http://pro.ganil-spiral2.eu/spiral2

17. A.H. Wuosmaa et al., Phys. Rev. C 72, 061301 (2005)

18. K.L.Jones et al., Nature 465, 454 (2010)

19. A.H. Wuosmaa et al., Nucl. Instr. Meth. A580, $1290(2007)$

20. J.C. Lighthall et al., Nucl. Instr. Meth. A622, 97 (2010)

21. H. Y. Lee et al., Phys. Rev. C 81, 015802 (2010)

22. B.B.Back et al., Phys. Rev. Lett. 104, 132501 (2010)

23. A.H. Wuosmaa et al., Phys. Rev. Lett. 105, 132501 (2010)

24. Z. Elekes et al., Phys. Lett. B586, 34 (2004)

25. N. Imai et al., Phys. Rev. Lett. 92, 062501 (2004)

26. M. Wiedeking et al., Phys. Rev. Lett. 100, 152501 (2008)

27. E.K. Warburton and B.A. Brown, Phys. Rev. C 46, 923 (1992) 